\title{
Impulsive aggressiveness of pregnant women affects the development of the fetal heart
}

\author{
Stefan Koelsch ${ }^{\text {a,*}}{ }$, Claudia Wiebigke ${ }^{\mathrm{b}}$, Walter A. Siebel ${ }^{\mathrm{c}}$, Holger Stepan ${ }^{\mathrm{d}}$ \\ a University of Sussex, Department of Psychology, Brighton, UK \\ b Max Planck Institute for Human Cognitive and Brain Sciences, Leipzig, Germany \\ c Conflict Research Center, Wiesbaden, Germany \\ d Department of Obstetrics, University of Leipzig, Germany
}

\section{A R T I C L E I N F O}

\section{Article history:}

Received 24 May 2009

Received in revised form 23 September 2009

Accepted 24 September 2009

Available online $\mathrm{xxxx}$

\section{Keywords:}

Fetal programming

Heart

mIED

Impulsive aggression

\begin{abstract}
A B S T R A C T
Mounting evidence indicates that the development of the fetus is heavily influenced by the intra-uterine milieu during pregnancy, and that such influence may have life-long consequences for the individual. The intra-uterine milieu is not only influenced by nutritional factors, but also by maternal endocrine and autonomic activity. Such activity is prone to be affected by an individual's personality, but only little is known about influences of maternal personality on the development of the fetus. We tested pregnant women for their propensity for impulsive, uncontrollable outbursts of temper (referred to here as moderate Intermittent Explosive Disorder, mIED). After the women gave birth, we measured electrocardiograms (ECGs) from their newborn infants to compare ECGs between newborns of women with and without mIED. The data show that infants of women with mIED have larger QRS complexes in the electrocardiogram, and lower heart rate variability, compared to infants of women without mIED. These results reveal effects of maternal mIED on the fetal heart development. These effects may predispose the individual to increased risk for later cardio-vascular disease. The findings open perspectives for better risk prevention models for the unborn child.
\end{abstract}

(C) 2009 Elsevier B.V. All rights reserved.

\section{Introduction}

About two decades ago, geographical as well as epidemiological studies provided evidence that health problems in adulthood like coronary heart disease and diabetes have origins related to intrauterine circumstances during fetal development (Barker and Osmond, 1986; Barker et al., 1989, 1993a,b). In the 1980s, Barker and coworkers (Barker and Osmond, 1986) first described the association between low birth weight and later death resulting from cardiovascular complications (also referred to as the Barker hypothesis; Barker and Osmond, 1986, De Boo and Harding, 2006), and this association was confirmed by a number of longitudinal studies (for a review see, e.g., De Boo and Harding, 2006). During the last years, an increasing number of experimental and epidemiological studies investigated the relation between sub-optimal intra-uterine environment (caused, e.g., by deficient nutrition or maternal stress), and increased risk for coronary heart disease, metabolic syndrome and type 2 diabetes (Curhan et al., 1996; Barker, 1998, 2002; Wadhwa 2005; Kajantie, 2006; Alexander, 2006; Jansson and Powell, 2007; Rondó et al., 2008).

\footnotetext{
* Corresponding author. Department of Psychology, Pevensey Building, University of Sussex, Falmer, Brighton, BN1 9QH, UK. Tel.: + 441273872776

E-mail address: koelsch@cbs.mpg.de (S. Koelsch).
}

According to current views, sub-optimal or even hostile intrauterine circumstances initiate a "fetal program" via altered cell differentiation, changed gene expression patterns, changed receptor expression, and epigenetic modifications that result in changes including metabolic, endocrine and hemodynamic parameters (for reviews, see Kajantie, 2006; Wadhwa, 2005; Seckl, 2008). Fetal programming serves the purpose of adapting to a sub-optimal intrauterine situation, and preparing for the future life ex utero. It occurs in response to different stressors such as under-nutrition, hypoxia, or increased glucocorticoid exposure in utero, and several studies have shown that such programming may have life-long consequences (Barker, 2002; Wadhwa, 2005; Kajantie, 2006; Lazinski et al., 2009; Lupien et al., 2009; Mastorci et al., 2009).

Recent research investigating the mechanisms underlying fetal programming has focussed on two systems that are activated during prenatal stress: the hypothalamic-pituitary-adrenal axis (HPA, which appears to be of major pathobiological importance for fetal programming effects; Lupien et al., 2009), and the autonomic nervous system (ANS, which interacts with the HPA and is also sensitive to stressful events during pregnancy). Exposure of a pregnant female to stress increases maternal glucocorticoid secretion (in particular the secretion of cortisol; for details see Seckl, 2008). Because these glucocorticoids pass in part through the placenta and reach the fetus, glucocorticoids also modulate the fetal HPA axis activity (e.g., 
Wadhwa, 2005). Numerous studies with animals have shown that fetal overexposure to stress hormones (glucocorticoids), either from maternal overproduction (in response to one or more stressors), or through exogenous administration, results in long-term stress-related endocrine and behavioural activity in the adult offspring (Barbazanges et al., 1996; Seckl, 2008).

In addition, endocrine activity of the fetus (including HPA axis activity), can also be modified by maternal nutrient restriction or maternal steroid action, which may result in long-term changes in the expression of steroid receptors within the fetus' limbic system (Weaver et al., 2004). Several studies in animals as well as in humans have identified that both the fetal HPA axis and the fetal reninangiotensin system are involved in the mediation of such neuroendocrine modifications (Langley-Evans, 2001; Alexander, 2006). The set-points of these endocrine systems can be altered in utero, and may alter HPA axis activity as well as autonomic function in later life (Alexander, 2006; Phillips and Jones, 2006).

Such alterations have been shown to predispose to hypertension and cardiometabolic stress (Drake et al., 2007), and thus to cardiovascular diseases. The heart is particularly vulnerable to intrauterine stressors, because (a) it can be affected in the embryo by hemodynamic forces that alter gene expression patterns in the wall of the heart, and (b) it can be affected in fetal life, in which cardiomyocytes undergo a maturational phase including endoreduplication of DNA or binucleation and escape from the cell cycle (Louey and Thornburg, 2005). However, long-term effects of prenatal stress have also been shown for other organs such as the pancreas (Chakravarthy et al., 2008), and in rats for a later increased responsiveness to stress and altered vessel reactivity to neuropeptide Y (Igosheva et al., 2007).

In humans, the linkage between the onset of an adaptive program before birth and consequences of this program after birth or even in adulthood has initially been investigated with regards to relations between body weight at delivery and later disorders like hypertension, metabolic syndrome and diabetes. During the last years, studies investigating effects of prenatal maternal depression, anxiety and psychological stress on the newborn have also demonstrated that the emotional state of a pregnant woman has an impact on the health and behaviour of the newborn. Well replicated evidence shows effects of maternal prenatal depression on the newborn, including higher norepinephrine levels (Lundy et al., 1996, 1999; Diego et al., 2004), higher cortisol and lower dopamine levels (Lundy et al., 1999; Diego et al., 2004; Field et al., 2004), as well as elevated heart rate (e.g., Allister et al., 2001; Field et al., 1995; Pickens and Field, 1995) and corresponding lower heart rate variability (Ponirakis et al., 1998) compared to children from mothers who were not depressed during pregnancy. Moreover, prenatal maternal depression is correlated with reduced fetal growth (see Field et al., 2006, for a review), as well as with reduced infant reactivity to facial expressions (Lundy et al., 1996) and to novel stimuli (Davis et al., 2007; for reviews see Wadhwa, 2005; Field et al., 2006). Notably, it was reported that effects of anxiety and depression during pregnancy on the reduction of birth weight and head size (as a measure of brain development) are comparable in magnitude to the effects of smoking during pregnancy (Lou et al., 1994).

The present study investigates whether fetal programming effects can be observed in infants from mothers with the propensity for impulsive, uncontrollable outbursts of temper, referred to here as moderate Intermittent Explosive Disorder (mIED; see also Koelsch et al., 2008; Koelsch, 2009). The phenomenology of mIED is derived from the DSM-IV-TR definition of Intermittent Explosive Disorder (IED), which is characterized by uncontrollable episodes of aggressive impulses that result in serious assaultive acts or destruction of property, and that are grossly out of proportion to the precipitating psychosocial stressor (e.g., McElroy, 1990; Coccaro et al., 1998; Olvera, 2002; Coccaro et al., 2007). IED is presumably more common than realized (with a lifetime prevalence of at least 5\% in the USA; Coccaro et al., 2004; Kessler et al., 2006) and thus represents an important cause of violent behaviour (e.g., McElroy, 1990; Coccaro et al., 1998, 2004, 2005; Kessler et al., 2006; Siever, 2008).

In contrast to IED, actions performed by individuals with mIED during impulsive episodes are, for the most part, judicially not relevant (e.g., door slamming, loud angry shouting, binge eating or binge drinking, smashing dishes etc.). Therefore, individuals with mIED do not fulfil the diagnostic criteria for IED, i.e., according to current diagnostic standards, mIED is not regarded as a disease, a psychopathology, or a personality disorder. Nevertheless, individuals with mIED often suffer from the disadvantageous impact of their temper tantrums on their family, their health, and their occupational career (e.g., McElroy, 1990; Coccaro et al., 1998; Koelsch et al., 2008).

A previous study (Koelsch et al., 2008) suggested that individuals with mIED are more agitated by, and show greater stress responses to sensory stimuli, consistent with the heightened central nervous arousal reported for impulsive aggression (Barratt and Slaughter, 1998), and with the restlessness and hyperactivity of some disorders characterized by substantial impulsivity (such as attention deficit/ hyperactivity disorder; Clarke et al., 2001). Moreover, although there are no data available on serotonin function in individuals with mIED, increased impulsivity has been implicated in low serotonin levels and altered HPA axis activity (Olvera, 2002; Clark et al., 2005), particularly with higher levels of glucocorticoids during impulsive episodes (Olvera, 2002). Based on animal (Barker, 2002; Langley-Evans, 2001) and human (Siebel and Winkler, 1996) models, this study aimed at testing whether such alterations influence the physiological development of the unborn child.

To investigate this, we classified pregnant women into groups with and without mIED (the classification was based on structured interviews and electroencephalographic data). In addition we obtained serum cortisol levels, and impulsivity scores using the impulsiveness scale of the $\mathrm{I}^{7}$ questionnaire (Eysenck et al., 1990). After the women gave birth, resting electrocardiograms (ECGs) of their newborn infants were measured; from these ECGs, we calculated (a) the heart rate variability (HRV) as a measure of autonomic cardiac activity, and (b) the $R-S$ wave amplitude (i.e., the QRS complex amplitude, see Supplemental Fig. 1 for illustration) as an index of cardiac dipole strength. Cardiac activity was examined because previous work established links between the intrauterine environment on the one side, and the development of the heart (Drake et al., 2007; Louey and Thornburg, 2005; Siebel and Winkler, 1996) as well as later cardiovascular disease on the other (Barker and Osmond, 1986). We hypothesized that $I^{7}$ scores and serum cortisol levels would differ between women with and without mIED, and that HRV and QRS complex amplitudes would differ between babies from women with and without mIED.

\section{Materials and methods}

\subsection{Study population}

From an initial sample of 52 individuals, 49 pregnant women (age range 22-39 years, mean age 27.8 years) without psychiatric disorders, as well as their newborn infants ( 22 boys and 27 girls, all born healthy at term) were included in the analysis. According to selfreports, none of the participants smoked, took drugs, or consumed alcohol during pregnancy. Pregnant women were measured in the third trimester (range was 27th to 39th gestational week). Participants were recruited in childbirth classes (using flyers and posters stating that pregnant women were invited to take part in a study on effects of increased impulsivity and temper tantrums on fetal development). Exclusion criteria were past or current diagnosis of a personality disorder, of an obsessive-compulsive or a major depressive disorder, past neurological illness or traumatic brain injury with 
hospital stay or coma. None of the subjects met DSM-IV criteria for borderline or antisocial personality disorder. Eight participants (five of them with $\mathrm{mIED}$ ) reported to have allergies, two (both without mIED) had hypothyreosis, one (also without mIED) hyperthyreosis, and two subjects (one with and one without mIED) reported to have migraine. Note that our groups of participants were thus not systematically biased with chronic disease, and that mIED did not confound with chronic disease.

27 women were classified to the control group, and 22 to the mIED group (the classification of subjects is described below). Age range was 20-40 years in both groups (mean age did not differ between groups, $p>.9$ ). The relatively high rate of mIED subjects in the study population is presumably due to the recruitment procedure (which stated that the objective of the study was to investigate effects of increased impulsivity and temper tantrums on fetal development), and thus not typical for women in general. Data on the incidence of mIED in the normal population are to date not available.

A written informed consent was obtained, the study was approved by the local ethics committee, and conducted according to the declaration of Helsinki.

\subsection{Women - classification of participants}

The classification of subjects into those with and without mIED was similar to a previous study (Koelsch, in press), and based on an electroencephalographic (EEG) measurement designed to assess mIED (Koelsch et al., 2008).

\subsection{EEG classification}

The EEG-based classification of pregnant mothers into those with and without mIED is described in the Supplemental methods. In brief, EEGs were recorded from participants during the presentation of auditory and visual stimulation. Then, twenty-three statistical values were calculated from the spectral EEG data (such as power values in the theta $[6-8 \mathrm{~Hz}]$ and the beta $[20-30 \mathrm{~Hz}]$ frequency bands). These values were entered into a model of standardized canonical discriminant function coefficients that were obtained in a previous study (Koelsch et al., 2008). The model returns a classification of each data set, that is, for each data set which was entered into the analysis, the model returns a probability for the group membership (with, without mIED) of this data set.

\subsection{Interviews}

For women who were assigned to the mIED group based on the EEG classification, the presence of mIED was checked based on a diagnostic interview (future studies should preferably conduct interviews blinded, and with all participants). Interviews were structured narrative interviews starting with the explanation that individuals differ with regard to how they experience and express anger, and that the purpose of the interview was to assess which kind of anger-type they are, followed by five questions: subjects were asked (1) whether they had over the course of the last months uncontrollable, impulsive outbursts of temper during which they shouted at other people, or attacked other people, or destroyed property, and - if so - (2) whether these actions were in excess of what they consider as appropriate with respect to the precipitating event. Participants were invited to give examples of anger episodes and to describe how they felt themselves before, during, and after such an episode. Furthermore, participants were asked (3) whether their temper outbursts first seemed to be switched on and then switched off, (4) whether they had regretted violent acts performed during an outburst immediately after the episode was over, and (5) whether such regret had not been helpful in averting such behaviour during subsequent episodes. Participants who answered all of these questions with "yes" remained in the mIED group (with regard to the third question, a common answer was that anger, or stress, builds up inside, and then suddenly discharges without being able to control it; typically, individuals with mIED also report that such discharges can be triggered even by relatively minor stressors). This was the case for 19 subjects. In addition, there were three subjects who met criteria for the implosive type of mIED (see next paragraph). Three other participants did not meet the criteria for mIED (despite the EEG classification); their data were not included in the study, resulting in the group size of 22 subjects with mIED.

Because some individuals with mIED may show auto-aggressive episodes (e.g., in extreme cases, self-injurious behaviour), rather than aggressive outbursts, subjects were also asked whether, over the course of the last months, they had experiences of uncontrollable inner rage during which they directed their aggression inwards, rather than outwards. With this regard, three subjects answered all five questions mentioned in the previous paragraph with "yes" and were, therefore, included in the mIED group (as in previous studies; Koelsch et al., 2008; Koelsch, in press).

Notably, all women with mIED reported that they can trace memories of impulsive-aggressive episodes back to their (early) childhood. Thus, it seems likely that all women of the mIED group had mIED before the onset of pregnancy (and that, thus, pregnancy was not the cause of their mIED).

Interviews were conducted in a separate session following the EEG session. Before the EEG recording session, participants also filled out the $\mathrm{I}^{7}$ questionnaire (Eysenck et al., 1990). After the EEG recording session, a blood sample was obtained for the measurement of cortisol from the first forty-two participants (twenty-one individuals from each group; cortisol was then determined using enzyme-linked immunosorbent assay). Because cortisol levels were virtually identical for both groups, no additional samples were drawn from the remaining seven participants. Due to difficulties in scheduling all participants for the same time of the day, we strived to match times of measurements between groups. However, due to the considerable intra-individual daytime variations in cortisol levels, future studies should obtain cortisol samples from all participants at the same time of the day.

\subsection{Infants - electrocardiographic (ECG) measurements}

The pregnant women were followed up to forty-two days after birth, at which point the ECG was recorded from their newborn infants. From each newborn infant, a standard 12-lead resting electrocardiogram (ECG) was obtained in supine position using PORTI-32/MREFA amplifiers (Twente Medical Systems BV) and a sampling rate of $1000 \mathrm{~Hz}$. ECGs were recorded for at least 2 min during awake state, but depending on how much the baby moved (which often caused movement artefacts), ECGs were recorded for up to $10 \mathrm{~min}$. For the calculation of the heart rate variability (HRV), time intervals between consecutive heart beats were computed (referred to as NN intervals, the time-point of a heart beat was defined by the fiducial point of the QRS complex, these points were automatically identified using the software package BIOSIG, http:// biosig.sourceforge.net, and controlled by the first author). From this time series, heart rate and RMSSD (square root of the mean squared differences of successive NN intervals) was calculated. RMSSD (instead of SDNN) was used as HRV index to compensate for different lengths of ECGs of babies (Malik et al., 1996). In addition to $\mathrm{NN}$ intervals, amplitudes of $R-S$ waves (i.e., QRS complex amplitudes, see Supplemental Fig. 1) were computed for each chest lead (by averaging all artefact-free $R-S$ waves of each lead) using the software Kardionoon 1.0 (www.kardionoon.net; see also Koelsch et al., 2007). All statistical tests were computed using SPSS 16.0 (SPSS Inc., Chicago, IL, USA). 


\section{Results}

\subsection{Women - behavioural and endocrinological data}

Impulsivity scores of the $\mathrm{I}^{7}$ questionnaire clearly differed between groups, providing reassurance that groups differed with regard to their impulsivity: As expected, scores were lower for the control group $(M=32.0, S E M=3.6)$ than for the mIED group $(M=46.1$, $S E M=5.2)$, the difference between groups being significant $(T(47)=$ $2.27, p<.03)$. Cortisol levels, however, did not differ between groups (controls: $M=556 \mathrm{nmol} / \mathrm{l}, S E M=30$; increased impulsivity: $M=$ $550 \mathrm{nmol} / \mathrm{l}, \mathrm{SEM}=21 ; p>.7)$.

\subsection{Women - electroencephalographical data}

Fig. 1 shows the spectral EEG data for the (upper) theta and the beta band (which were used for the classification of subjects, for details see Supplemental material). Power values differed between the two groups (Fig. 1A, B), during both auditory and visual stimulation: As expected, power values of oscillations elicited at centro-parietal electrodes in the (upper) theta band were lower in the mIED group than in the control group (Fig. 1A). By contrast, power values at frontal and fronto-temporal electrodes in the beta band were higher in the mIED group than in the control group (Fig. 1B).

These observations were statistically confirmed: An ANOVA of the power values of the EEG data recorded during auditory and visual stimulation at centro-parietal electrodes in the upper theta band $(7-8 \mathrm{~Hz})$ with factors group (control, mIED), stimulus (auditory, visual) and region of interest (left, middle, right) indicated an effect of group $(F(1,47)=4.03$, $p<.05$, no two- or three-way interactions). An ANOVA for the beta band $(20-30 \mathrm{~Hz})$ with factors group, stimulus, and hemisphere also indicated an effect of group $(F(1,47)=4.02, p=.05)$, and an effect of stimulus $(F$ $(1,47)=8.19, p<.01$, reflecting that group differences were stronger during the visual condition). The slight right-hemispheric weighting of the group difference in beta activity observable in Fig. 1B was statistically not significant $(p=.15$ for the interaction between factors group and hemisphere). Results of the ANOVAs show that brain activity differed between groups in both the upper theta and in the beta band. No significant differences between groups were found in the lower $(8-10 \mathrm{~Hz})$ or upper $(10-12 \mathrm{~Hz})$ alpha band $(p>.2$ in each test).

\subsection{Infants - electrocardiographical data}

In the ECGs of infants, differences between children from women with mIED, and from women of the control group were observed in both the heart rate variability (HRV) and the amplitude pattern of the infants' ECGs: infants from women with mIED had a lower HRV (as indicated by a lower RMSSD, see Table 1 and Fig. 2A), and the $R-S$ wave amplitudes were larger in this group of infants (Figs. 2B and 3). Differences in HRV appeared to be due to differences in both the lowfrequency and the high-frequency power, which were both nominally lower in the group of infants from women with mIED (these differences were, however, statistically not significant, Table 1a). Group differences in $R-S$ amplitudes were largest for electrodes situated around the apex of the heart (V3-V6), but also clearly observable when calculating the mean of the $R-S$ waves measured at all chest leads (V1-V6), or at the chest lead with the largest $R-S$ amplitude (Table 1). A 2-way univariate ANOVA with the $R-S$ amplitude values of V3-V6 (averaged separately for each individual across these four electrodes) as dependent variable, mIED of mothers as well as gender of newborns as fixed factors, and body weight as covariate, indicated a significant effect of $\operatorname{mIED}(F(1,4)=10.48$, $p<.002)$. There was no effect of gender $(F(1,4)=.4, p=.53)$ or weight $(F(1,4)=.7, p=.41)$, and no interaction effect $(F(1,4)=.8, p=.77)$, indicating that mIED of mothers, but neither gender nor body weight of the newborns had a significant effect on the $R-S$ wave amplitude (i.e., on the amplitude of the QRS complex). Essentially the same results were obtained with an analogous ANOVA of the $R-S$ amplitude values of the chest lead with the maximal $R-S$ amplitude, and with an ANOVA of the $R-S$ values of all chest leads (V1-V6) as dependent variables: effects were observed for factor mIED (chest lead with maximal $R-S$ amplitude: $p<.05$; mean $R-S$ amplitude of all chest leads: $p<.02$ ), with no further effects or interactions. Note that group differences were significant when calculating the $R-S$ amplitude for

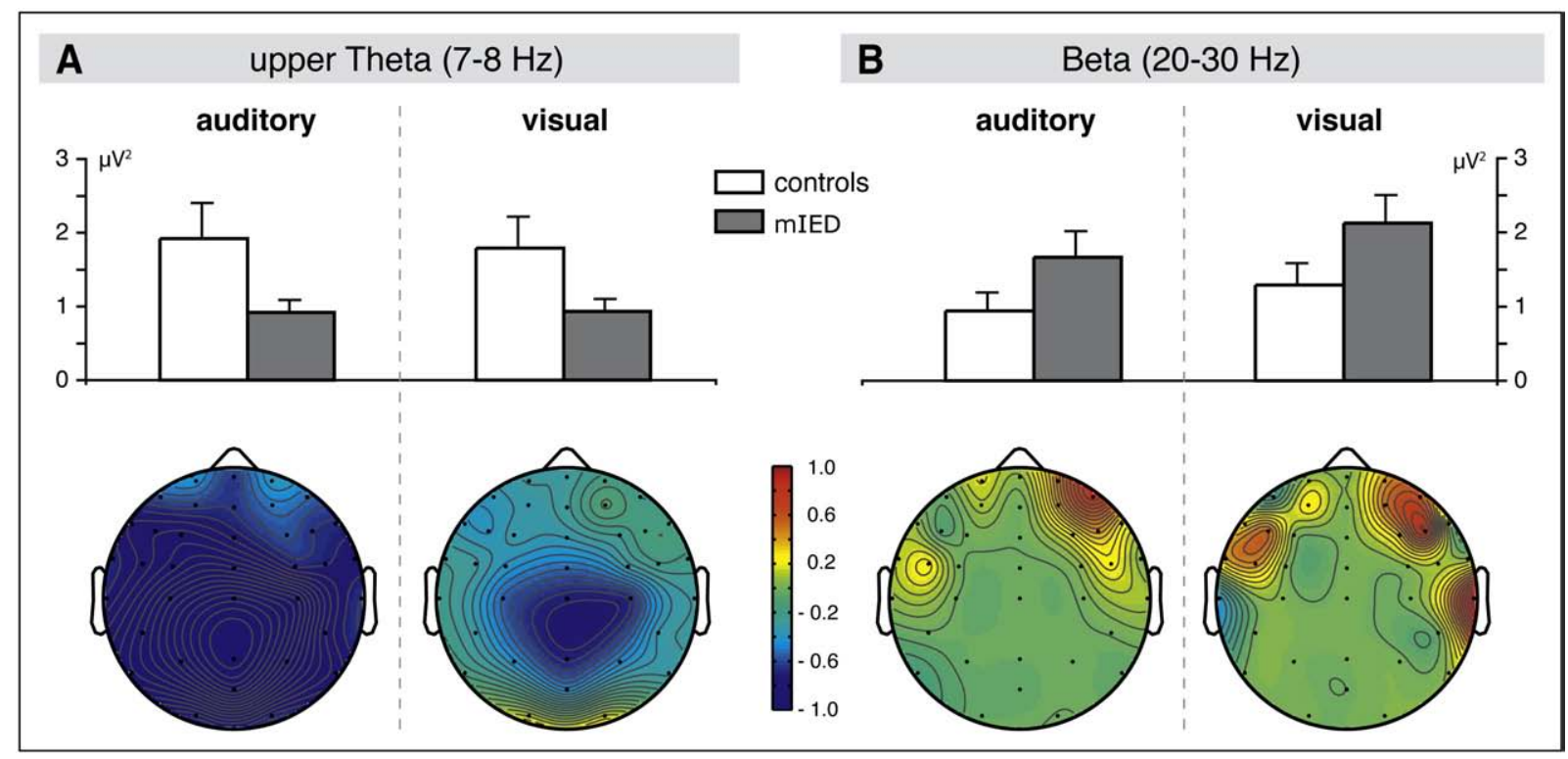

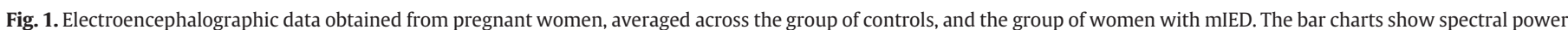

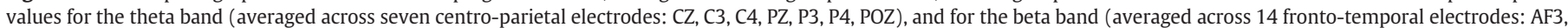

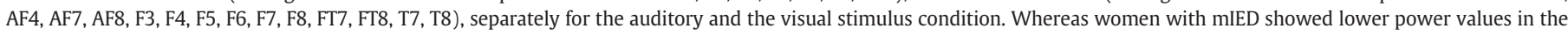

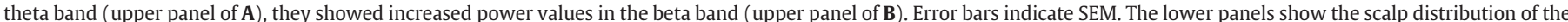

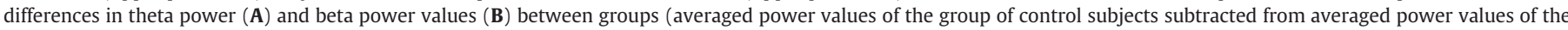

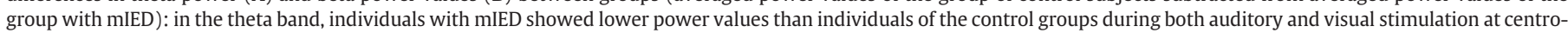
parietal electrodes. In the beta band, power values in the beta band were larger in the impulsive (mIED) group than in the control group at fronto-temporal electrode sites. 
Table 1

Infant data

\begin{tabular}{lccl}
\hline & Mothers controls & $\begin{array}{l}\text { Mothers with increased } \\
\text { impulsivity }\end{array}$ & $\begin{array}{l}\text { Group difference } \\
(p \text {-values })\end{array}$ \\
\hline NN (ms) & $408.31(8.55)$ & $400.18(6.12)$ & .46 \\
RMSSD (ms) & $9.49(.7)$ & $7.70(.54)$ & .05 \\
LF & $8.37(1.2)$ & $6.22(.69)$ & .12 \\
HF & $2.30(.035)$ & $1.58(.32)$ & .14 \\
$R-S_{\text {max }}(\mathrm{mV})$ & $3.082(128.5)$ & $3.606(162.8)$ & .02 \\
$R-S_{\text {V3-v6 }}(\mathrm{mV})$ & $2173(75.9)$ & $2662(95)$ & .0002 \\
Birth length $(\mathrm{cm})$ & $50.5(.38)$ & $51.1(.40)$ & .24 \\
Birth weight $(\mathrm{g})$ & $3506.9(71.8)$ & $3713.4(81.9)$ & .06 \\
Ponderal index & $30.1(.17)$ & $30.2(.16)$ & .54 \\
Gestational age & $39.7(.15)$ & $39.8(.15)$ & .90 \\
\hline
\end{tabular}

$\mathrm{NN}$ : time intervals between subsequent heart beats; RMSSD: root mean square of standard deviation of inter-heartbeat intervals; LF: low frequency power of heart rate variability $(0.05-0.25 \mathrm{~Hz})$; HF: high frequency power of heart rate variability $(0.25-$ $1 \mathrm{~Hz}) ; R-S_{\max }: R-S$ amplitudes obtained from the chest lead with the largest $R-S$ amplitude; $R-S_{\mathrm{V} 3-\mathrm{V} 5}: R-S$ amplitude values of chest leads V3-V6 (averaged separately for each individual across these four electrodes). Values are provided separately for infants of women with and without mIED, all values represent group means (with standard errors provided in parentheses).

each individual's chest lead with the maximal $R-S$ amplitude, rendering it implausible that amplitude differences between infant groups were simply due to differences in heart orientation.

Infant groups did not differ with regards to heart rate, birth length, Ponderal index, or gestational age (Table 1); the weight was nominally slightly higher for babies from mIED mothers, but this difference between groups was statistically only marginally significant $(p<.06$; Table 1$)$.

\subsection{Correlations between data from infants and mothers}

We also performed a linear regression with discriminant scores of women (on the basis of which they were assigned to either of the two groups), and $R-S$ amplitudes of their children as variables. This regression indicated a moderate, but significant relationship between the two variables $\left(r^{2}=.3, p<.0001\right.$; see Fig. 4$)$.

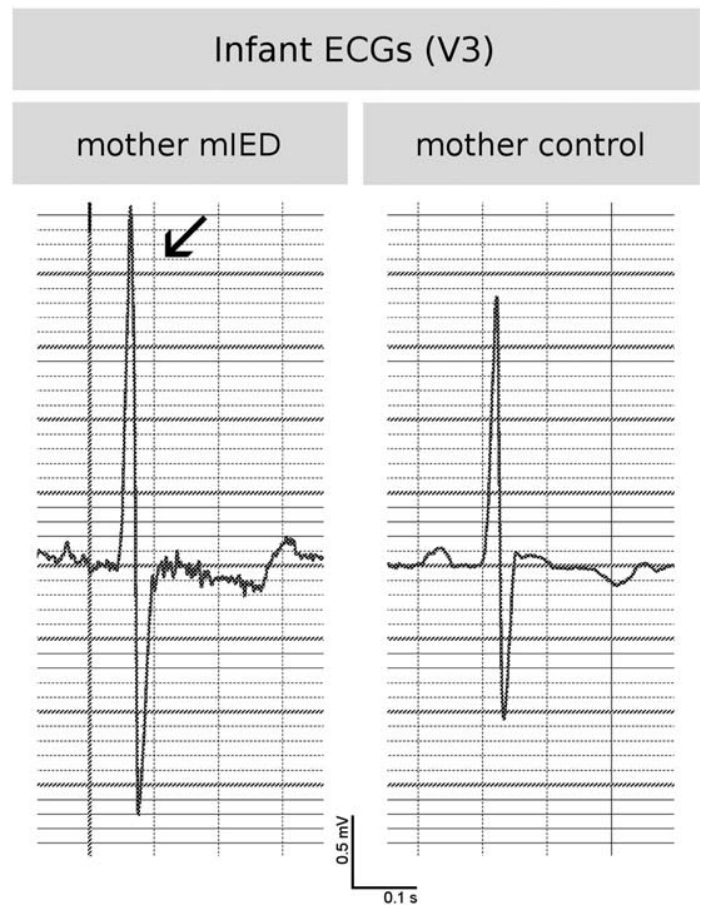

Fig. 3. Illustration of infant ECGs (recorded from the chest lead V3), separately for an infant from a mother with (left panel) and without mIED (right panel). Note the difference in $R-S$ wave amplitudes (indicated by the arrow).

No correlations were observed between $R-S$ amplitudes of mothers and their infants, neither with regard to the $R-S$ amplitude values averaged across V3-V6, nor with regard to $R-S$ amplitude values obtained from the chest lead with the maximal $R-S$ amplitude $\left(r^{2}<.02, p>.4\right.$ in both tests). Likewise, no correlations were found between HRV measures of mothers and infants, neither with regard to RMSSD, nor with regard to SDNN $\left(r^{2}<.02, p>.3\right.$ in both tests). In contrast to the ECG data from infants, $R-S$ amplitudes of adults did not differ between individuals with and without mIED, neither with

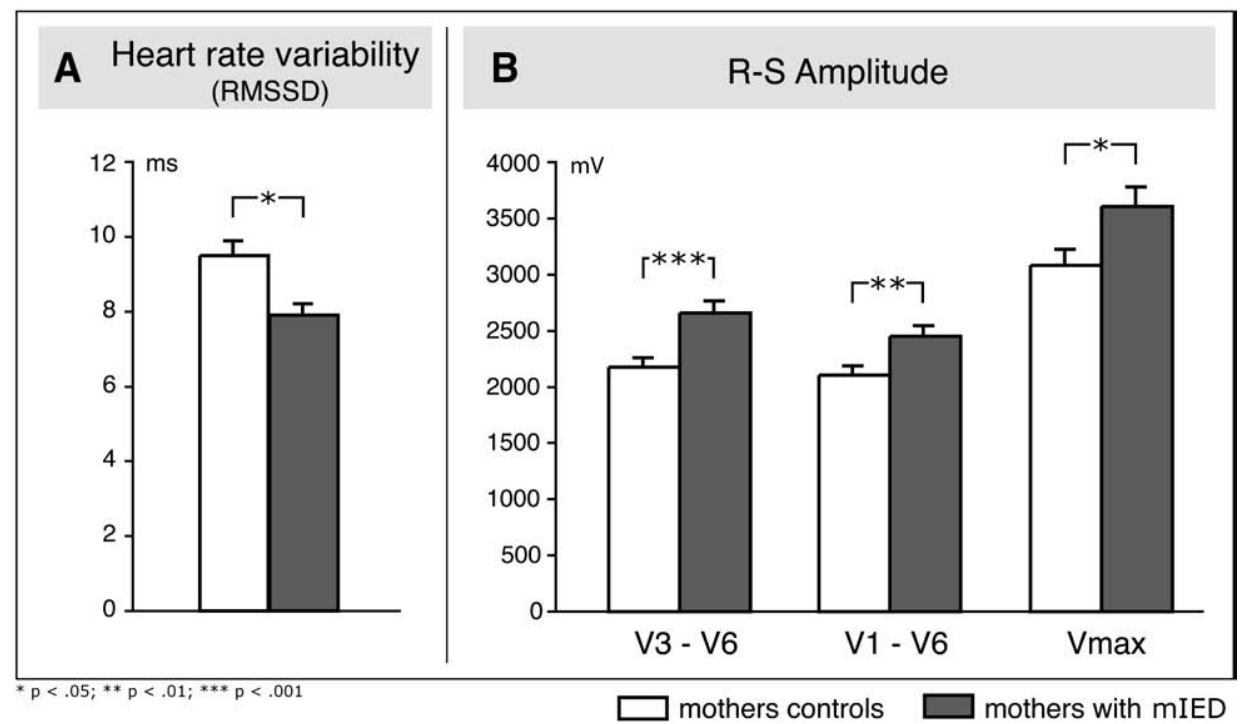

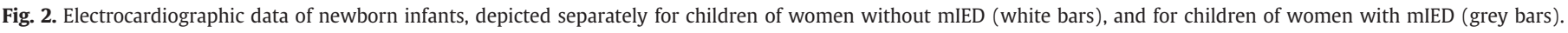

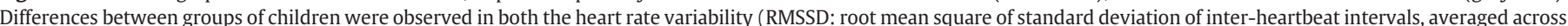

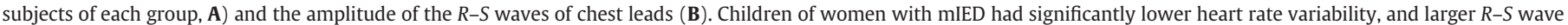

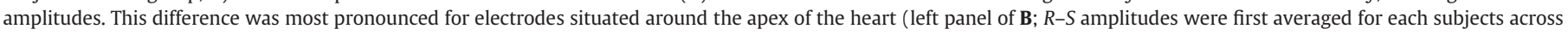

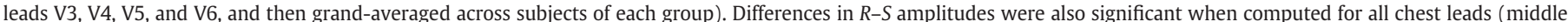

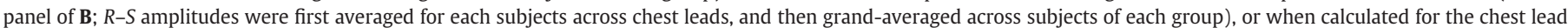

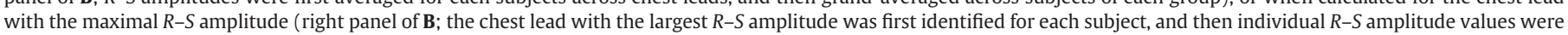
grand-averaged across subjects of each group). Error bars indicate SEM. 


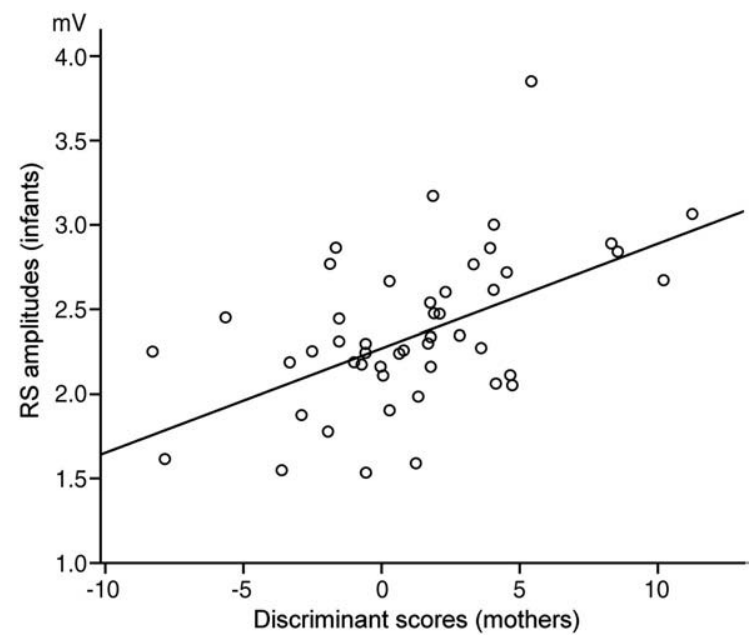

Fig. 4. Linear regression performed with discriminant scores of women, and $R-S$ amplitudes of their newborn infants (mean of leads V3-V6) as variables. Note that the discriminant scores were used to assign women to either of the two groups (higher scores are related to increased impulsiveness; one data set was excluded from the analysis because of an extreme discriminant score).

regard to the $R-S$ amplitude values averaged across V3-V6, nor with regard to $R-S$ amplitude values obtained from the chest lead with the maximal $R-S$ amplitude ( $p>.25$ in both tests). Likewise, neither heart rate, nor RMSSD or SDNN differed between adults with and without mIED ( $p>.2$ in all tests).

\section{Discussion}

HRV was lower, and amplitudes of $R-S$ waves larger, in newborn infants of women with mIED compared to newborns of women without mIED. This reveals that the propensity for impulsive, uncontrollable outbursts of temper in pregnant women has effects on the physiological development of the unborn child. HRV has been established as an index of autonomic nervous system (ANS) activity (Malik et al., 1996). Therefore, our results indicate that maternal personality can exert effects on the ANS activity of the unborn child during pregnancy, and that these effects are clearly observable even after birth. The finding of reduced HRV in infants of women with mIED is important because reduced HRV is discussed as a marker of increased risk for cardiovascular disease (Malik et al., 1996; Tsuji et al., 1996; Martin and Krum, 2007; Nuyt, 2008). Therefore, the present data suggest that, during pregnancy, maternal mIED generates an increased risk in the child for later cardiac vulnerability. This opens future perspectives for further risk prevention for cardiovascular disease, for example by offering therapeutic treatment to women with mIED before pregnancy. Differences between infant groups were only observed in the temporal parameters of the HRV (namely, the RMSSD), not in spectral parameters (low or high frequency measures). The lack of significant differences in the spectral parameters is presumably due to the fact that we obtained relatively short ECGs ( $2 \mathrm{~min})$ from the infants, resulting in a low signal-tonoise ratio; for future studies it thus appears recommendable to record infant ECGs over longer time periods.

In addition to the differences in HRV, cardiac activity also differed with regards to the amplitudes of $R-S$ waves (i.e., QRS complex amplitudes), which were larger in the group of babies of women with mIED, independent of whether they were measured at electrodes situated around the apex of the heart, at all chest leads, or at the chest lead with the maximal $R-S$ amplitude. This renders it implausible that simply heart orientation was responsible for these differences between groups. Moreover, it is unlikely that simply differences in tissue conductivity account for the differences in QRS complex amplitudes between groups, because (a) both groups did statistically not differ with regards to birth length, and (b) birth weight was nominally even higher in the infants of women with mIED. Therefore, the present data indicate that the differences in $R-S$ amplitudes reflect a difference in the development of the heart between groups. The larger QRS complex amplitudes in the infants of women with mIED perhaps reflect an adaptation to increased intra-uterine glucocorticoid levels (which require an increased stroke volume to compensate for reduced oxygen levels during impulsive episodes, see also Wadhwa, 2005), but whether this difference in amplitudes is due to the growth of cardiomyocytes, or due to altered signal transduction mechanisms, remains to be specified.

The $R-S$ amplitudes of newborns correlated with the discriminant scores of mothers (these scores were used for the classification into mothers with and without mIED, and are thus indicative of impulsive aggressiveness). At the same time, $R-S$ amplitudes of mothers did not correlate with mIED (indicating that enlarged $R-S$ amplitudes are not related to mIED of an individual), and there was no correlation between the $R-S$ amplitudes of mothers and the $R-S$ amplitudes of their infants (arguing against a mere hereditary component). These findings underline that mIED has an effect on the fetal development of the heart.

Women with mIED showed lower power values in the theta band, along with increased power values in the beta band. This spectral pattern has previously been related to increased HPA axis activity (Chapotot et al., 1998; Koelsch et al., 2008), and fits well with previous research showing alterations of central neurotransmitter (decreased serotonin), and increased HPA axis activity in individuals with increased impulsivity (e.g., Olvera, 2002). Although cortisol levels did not differ between groups of women in the present study, it is likely that impulsive-aggressive episodes are accompanied by both increased sympathetic and increased HPA axis activity. Previous research in animals strongly suggests that such hormonal alterations have effects on the physiological development of the fetus' cardiovascular system (Meyer and Zhang, 2007). Note that the hormonal changes that occur during impulsive episodes are likely to return to normal after such episodes, perhaps explaining in part why cortisol levels did not differ between groups in the present study. The exact mechanisms of such endocrine alterations, thus, remain to be investigated. Future studies could benefit from obtaining several cortisol samples at the same time of the day, over the course of several days, to attain more representative cortisol level values.

Interestingly, a recent study (Huh et al., 2008) showed that increased glucocorticoid levels in umbilical cord blood were directly associated with higher offspring systolic blood pressure at age 3. Although not directly shown in that study (Huh et al., 2008), it seems likely that the increased glucocorticoid levels are indicative of higher stress levels during pregnancy, and that the increased levels of stress hormones during pregnancy led to increased systolic BP in the children. This is consistent with our findings which suggest that increased HPA axis activity in pregnant women with mIED (e.g. as reflected in the increased beta power values) has effects on fetal heart development.

Limitations of the present study include the relatively small number of subjects, and future studies should replicate the present findings with larger sample sizes. Such studies should also measure blood pressure (and perhaps determine cortisol/cortisone ratios in umbilical cord blood), and aim to elucidate the pathophysiological mechanisms that link maternal mIED with fetal cardio-vascular alterations, preferably with the help of more detailed endocrinological data. Finally, long-term studies should investigate about how long the heart alterations seen in newborns persist.

\section{Conclusions}

The present study provides the first empirical evidence that mIED in pregnant women has effects on the development of the fetal heart. Such effects may have life-long consequences for organ activity, autonomic activity, and disease susceptibility. Specifically, due to the 
association between lower heart rate variability and increased cardiac vulnerability, maternal mIED might pose an immediate risk for later cardio-vascular disease. Because impulsive aggressiveness is presumably more common than realized, our findings open important perspectives for future research on the prevention of cardiac disease even before birth. Finally, the present study shows that the exciting theory of fetal programming has to be expanded to include - beyond intrauterine nutrition - also parameters of maternal psyche and personality to develop better risk prevention models for the unborn child.

\section{Acknowledgement}

We thank Sylvia Stasch for her valuable help in scheduling and measuring subjects.

\section{Appendix A. Supplementary data}

Supplementary data associated with this article can be found, in the online version, at doi:10.1016/j.ijpsycho.2009.09.008.

\section{References}

Alexander, B.T., 2006. Fetal programming of hypertension. Am. J. Physiol. Regul. Integr Comp. Physiol. 290 (1), R1-R10.

Allister, L., Lester, B.M., Carr, S., Liu, J., 2001. The effects of maternal depression on fetal heart rate response to vibroacoustic stimulation. Dev. Neuropsychol. 20 (3) 639-651.

Barbazanges, A., Piazza, P.V., Le Moal, M., Maccari, S., 1996. Maternal glucocorticoid secretion mediates long-term effects of prenatal stress. J. Neurosci. 16, 3943-3949.

Barker, D.J.P., 1998. In utero programming of chronic disease. Clin. Sci. 95, 115-128.

Barker, D.J., 2002. Fetal programming of coronary heart disease. Trends Endocrinol. Metab. 13 (9), 364-368.

Barker, D.J., Osmond, C., 1986. Infant mortality, childhood nutrition, and ischaemic heart disease in England and Wales. Lancet 8489, 1077-1081.

Barker, D.J., Winter, P.D., Osmond, C., Margetts, B., Simmonds, S.J., 1989. Weight in infancy and death from ischaemic heart disease. Lancet 2, 577-580.

Barker, D.J., Hales, C.N., Fall, C.H., Osmond, C., Phipps, K., Clark, P.M., 1993a. Type 2 (noninsulin-dependent) diabetes mellitus, hypertension and hyperlipidaemia (syndrome X): relation to reduced fetal growth. Diabetologia 36, 62-67.

Barker, D.J., Osmond, C., Simmonds, S.J., Wield, G.A., 1993b. The relation of small head circumference and thinness at birth to death from cardiovascular disease in adult life. Br. Med. J. 306, 422-426.

Barratt, E.S., Slaughter, L., 1998. Defining, measuring, and predicting impulsive aggression: a heuristic model. Behav. Sci. Law. 16 (3), 285-302.

Chakravarthy, M.V., Zhu, Y., Wice, M.B., Coleman, T., Pappan, K.L., Marshall, C.A., McDaniel, M L., Semenkovich, C.F., 2008. Decreased fetal size is associated with beta-cell hyperfunction in early life and failure with age. Diabetes 57 (10), 2698-2707.

Chapotot, F., Gronfier, C., Jouny, C., Muzet, A., Brandenberger, G., 1998. Cortisol secretion is related to electroencephalographic alertness in human subjects during daytime wakefulness. J. Clin. Endocrin. Metab. 83, 4263-4268.

Clark, L., Roiser, J.P., Cools, R., Rubinsztein, D.C., Sahakian, B.J., Robbins, T.W., 2005. Stop signal response inhibition is not modulated by tryptophan depletion or the serotonin transporter polymorphism in healthy volunteers: implications for the 5 HT theory of impulsivity. Psychopharmacology 182 (4), 570-578.

Clarke, A.R., Barry, R., McCarthy, R., Selikowitz, M., 2001. Excess beta activity in children with attention-deficit/hyperactivity disorder: an atypical electrophysiologica group. Psych. Res. 103 (2-3), 205-218.

Coccaro, E.F., Kavoussi, R.J., Berman, M.E., Lish, J., 1998. Intermittent explosive disorderrevised: development, reliability, and validity of research criteria. Compr. Psychiatry 39 (6), 368-376.

Coccaro, E.F., Schmidt, C.A., Samuels, J.F., Nestadt, G., 2004. Lifetime and 1-month prevalence rates of intermittent explosive disorder in a community sample. J. Clin. Psychiatry 65, 820-824.

Coccaro, E.F., Posternak, M.A., Zimmerman, M., 2005. Prevalence and features of intermittent explosive disorder in a clinical setting. J. Clin. Psychiatry 66 (10) $1221-1227$

Coccaro, E.F., McCloskey, M.S., Fitzgerald, D.A., Phan, K.L., 2007. Amygdala and orbitofrontal reactivity to social threat in individuals with impulsive aggression. Biol. Psychiatry 62 (2), 168-178.

Curhan, G.C., Willett, W.C., Rimm, E.B., et al., 1996. Birth weight and adult hypertension, diabetes mellitus, and obesity in US men. Circulation 94, 3246-3250.

Davis, E.P., Glynn, L.M., Schetter, C.D., Hobel, C., Chicz-Demet, A., Sandman, C.A., 2007. Prenatal exposure to maternal depression and cortisol influences infant temperament. J. Am. Acad. Child Adolesc. Psych. 46 (6), 737-746.

De Boo, H.A., Harding, J.E., 2006. The developmental origins of adult disease (Barker) hypothesis. Aust. N. Z. J. Obstet. Gynaecol. 46 (1), 4-14.

Diego, M.A., Field, T., Hernandez-Reif, M., Cullen, C., Schanberg, S., Kuhn, C., 2004. Prepartum, postpartum, and chronic depression effects on newborns. Psychiatry 67 (1), 63-80.
Drake, A.J., Tang, J.I., Nyirenda, M.J., 2007. Mechanisms underlying the role of glucocorticoids in the early life programming of adult disease. Clin. Sci. (Lond.) 113 (5), 219-232.

Eysenck, S.G., Daum, I., Schugens, M.M., Diehl, J.M., 1990. A cross-cultural study of impulsiveness, venturesomeness and empathy: Germany and England. Diff. Diagn. Psych. 11 (4), 209-213.

Field, T., Pickens, J., Fox, N.A., Nawrocki, T., 1995. Vagal tone in infants of depressed mothers. Dev. Psychopathol. 7 (2), 227-231.

Field, T., Diego, M., Dieter, J., Hernandez-Reif, M., Schanberg, S., Kuhn, C., Yando, R., Bendell, D., 2004. Prenatal depression effects on the fetus and the newborn. Infant Behav. Dev. 27, 216-229.

Field, T., Diego, M., Hernandez-Reif, M., 2006. Prenatal depression effects on the fetus and newborn: a review. Infant Behav. Dev. 29 (3), 445-455.

Huh, S.Y., Andrew, R., Rich-Edwards, J.W., Kleinman, K.P., Seckl, J.R., Gillman, M.W., 2008. Association between umbilical cord glucocorticoids and blood pressure at age 3 years. BMC Med. 6, 25.

Igosheva, N., Taylor, P.D., Poston, L., Glover, V., 2007. Prenatal stress in the rat results in increased blood pressure responsiveness to stress and enhanced arterial reactivity to neuropeptide $\mathrm{Y}$ in adulthood. J. Physiol. 582, 665-674.

Jansson, T., Powell, T.L., 2007. Role of the placenta in fetal programming: underlying mechanisms and potential interventional approaches. Clin. Sci. 113, 1-13.

Kajantie, E., 2006. Fetal origins of stress-related adult disease. Ann. N. Y. Acad. Sci. 1083, $11-27$.

Kessler, R.C., Coccaro, E.F., Fava, M.F., Jaeger, S., Jin, R., Walters, E., 2006. The prevalence and correlates of DSM-IV intermittent explosive disorder in the national comorbidity survey replication. Arch. Gen. Psychiatry 63, 669-678.

Koelsch, S., Remppis, A., Sammler, D., Jentschke, S., Mietchen, D., Fritz, T., Bonnemeier, H., Siebel, W.A., 2007. A cardiac signature of emotionality. Eur. J. Neurosci. 26, 3328-3338.

Koelsch, S., Sammler, D., Jentschke, S., Siebel, W.A., 2008. EEG correlates of moderate intermittent explosive disorder. Clin. Neurophysiol. 119, 151-162.

Koelsch, S., 2009. P3a and mismatch negativity in individuals with moderate Intermittent Explosive Disorder. Neurosci Lett. 460 (1), 21-26.

Langley-Evans, S.C., 2001. Fetal programming of cardiovascular function through exposure to maternal undernutrition. Proc. Nutr. Soc. 60 (4), 505-513.

Lazinski, M.J., Shea, A.K., Steiner, M., 2009. Effects of maternal prenatal stress on offspring development: a commentary. Arch. Womens Health 11, 363-375.

Lou, H.C., Hansen, D., Nordentoft, M., Pryds, O., Jensen, F., Nim, J., Hemmingsen, R., 1994. Prenatal stressors of human life affect fetal brain development. Dev. Med. Child Neurol. 36 (9), 826-832.

Louey, S., Thornburg, K.L., 2005. The prenatal environment and later cardiovascular disease. Early Hum. Dev. 81, 745-751.

Lundy, B., Field, T., Pickens, J., 1996. Newborns of mothers with depressive symptoms are less expressive. Infant Behav. Dev. 19 (4), 419-424.

Lundy, B.L., Jones, N.A., Field, T., Nearing, G., Davalos, M., Pietro, P., Schanberg, S., Kuhn, C., 1999. Prenatal depression effects on neonates. Infant Behav. Dev. 22 (1), 119-129.

Lupien, S.J., McEwen, B.S., Gunnar, M.R., Heim, C., 2009. Effects of stress throughout the lifespan on the brain, behaviour and cognition. Nat. Rev., Neurosci. 10 (6), 434-445.

Malik, M., et al., 1996. Heart rate variability: standards of measurement, physiological interpretation and clinical use. Eur. Heart J. 17, 354-381.

Martin, J.H., Krum, H., 2007. Statins and clinical outcomes in heart failure. Clin. Sci. 113 (3), 119-127.

Mastorci, F., Vicentini, M., Viltart, O., Manghi, M., Graiani, G., Quaini, F., Meerlo, P., Nalivaiko, E., Maccari, S., Sgoifo, A., 2009. Long-term, effects of prenatal stress: changes in adult cardiovascular regulation and sensitivity to stress. Neurosci. Biobehav. Rev. 33 (2), 191-203.

McElroy, S.L., 1990. Recognition and treatment of DSM-IV intermittent explosive disorder. J. Clin. Psychol, 60 (Suppl 15), 12-16.

Meyer, K., Zhang, L., 2007. Fetal programming of cardiac function and disease. Reprod. Sci. 14 (3), 209-216.

Nuyt, A.M., 2008. Mechanisms underlying developmental programming of elevated blood pressure and vascular dysfunction: evidence from human studies and experimental animal models. Clin. Sci. 114 (1), 1-17.

Olvera, R.L., 2002. Intermittent explosive disorder. CNS Drugs 16 (8), 517-526.

Phillips, D.I., Jones, A., 2006. Fetal programming of autonomic and HPA function: do people who were small babies have enhanced stress responses? J. Physiol. 572 (Pt 1), 45-50.

Pickens, J.N., Field, T., 1995. Facial expressions and vagal tone of infants of depressed and non-depressed mothers. Early Dev. Parent. 4 (2), 83-89.

Ponirakis, A., Susman, E.J., Stifter, C.A., 1998. Negative emotionality and cortisol during adolescent pregnancy and its effects on infant health and autonomic nervous system reactivity. Dev. Psychobiol. 33 (2), 163-174.

Rondó, P.H.C., Lemos, J.O., Pereira, J.A., Oliveira, J.M., Innocente, L.R., 2008. Relationship between birth weight and arterial elasticity in childhood. Clin. Sci. do1:10.1042/ CS20070420

Seckl, J.R., 2008. Glucocorticoids, developmental 'programming' and the risk of affective dysfunction. Prog. Brain Res. 167, 17-34.

Siebel, W.A., Winkler, T., 1996. Noosomatik Vol. V, 2nd ed. Glaser, Langwedel.

Siever, L.J., 2008. Neurobiology of aggression and violence. Am. J. Psychiatry 165 (4), 429-442.

Tsuji, H., Larson, M.G., Venditti Jr, F.J., Manders, E.S., Evans, J.C., Feldman, C.L., Levy, D., 1996. Impact of reduced heart rate variability on risk for cardiac events. The Framingham Heart Study. Circulation 94 (11), 2850-2855.

Wadhwa, P.D., 2005. Psychoneuroendocrine processes in human pregnancy influence fetal development and health. Psychoneuroendocrinology 30 (8), 724-743.

Weaver, I.C., Cervoni, N., Champagne, F.A., D'Alessio, A.C., Sharma, S., Seckl, J.R., Dymov, S., Szyf, M., Meaney, M.J., 2004. Epigenetic programming by maternal behavior. Nat. Neurosci. 7 (8), 847-854. 